SFB/CPP-11-45

TTP11-24

\title{
Gluino-Squark Production at the LHC: The Threshold
}

\author{
Matthias R. Kauth, Achim Kress and Johann H. Kühn \\ Institut für Theoretische Teilchenphysik \\ Karlsruhe Institute of Technology (KIT) \\ 76128 Karlsruhe, Germany
}

\begin{abstract}
An analysis of the cross section for hadronic production of gluino-squark pairs close to threshold is presented. Within the framework of non-relativistic QCD a significant enhancement compared to fixed order perturbation theory is observed which originates from the characteristic remnants of the gluino-squark resonances below the nominal pair threshold. The analysis includes all colour configurations of $S$-wave gluino-squark pairs, i.e. triplet, sextet and $\mathbf{1 5}$ representation. Matching coefficients at leading order are separately evaluated for all colour configurations. The dominant QCD corrections, arising from initial- and final-state radiation are included. The non-relativistic dynamics of the gluino pair is solved by calculating the Green's function in Next-to-Leading Order (NLO). The results are applied to benchmark scenarios, based on Snowmass Points and Slopes (SPS). As a consequence of the large decay rate of at least one of the constituents (squark or gluino), annihilation decays of the bound state $(\tilde{g} \tilde{q}) \rightarrow g q, q \gamma, q Z$ or $q^{\prime} W^{ \pm}$are irrelevant. Thus the signatures of gluino-quark production below and above the nominal threshold are identical. Numerical results for the cross section at the Large Hadron Collider (LHC) at $\sqrt{s}=7 \mathrm{TeV}$ and $14 \mathrm{TeV}$ are presented. The enhancement of the total cross section through final state interaction amounts to roughly $3 \%$.
\end{abstract}




\section{Introduction}

The search for new particles, predicted in supersymmetric models, is one of the most important tasks of the experiments at the LHC. The detailed determination of particle masses and couplings will be crucial for the discrimination between various manifestations of supersymmetry (SUSY) and alternative models, even more so if one wants to distinguish between the different variants of supersymmetric models, to identify the origin of breaking of supersymmetry and to measure the model parameters. One of the SUSY signals will be events with missing energy or missing transverse momentum, resulting from cascade decays of squarks and gluinos into the lightest supersymmetric particle (LSP) which escapes detection. The existence of squarks, gluinos and the LSP is definitely a key prediction of supersymmetry.

Depending on details of the models, in particular the mass of the LSP, gluinos and squarks with masses up to $3 \mathrm{TeV}$ [1] could be detected. The decay rates of gluinos and squarks depend strongly on the ratio of squark and gluino masses. If the gluino is heavier $\left(m_{\tilde{g}}>\right.$ $m_{\tilde{q}}$ ), the two-body decay of the gluino into a squark and an antiquark (or its charge conjugate) is possible with the squark decaying into a quark plus a chargino or neutralino. In the opposite case $\left(m_{\tilde{g}}<m_{\tilde{q}}\right)$ the two-body decay of the gluino is kinematically forbidden and the now dominant three-body decay into quark, antiquark and neutralino or chargino, mediated by the virtual squark, leads to a small decay rate for the gluino while the squark decays into the gluino plus a quark.

The importance of squark and gluino searches has motivated a series of detailed studies of hadroproduction cross sections for squarks and gluinos. The lowest order has been evaluated long time ago [2 4]. Subsequently NLO SUSY-QCD corrections were calculated [5] 7], more recently the effect of soft-gluon resummation [8 14] was included. The present paper will be concerned with gluino-squark-pair production close to threshold, which exhibits a number of peculiar features.

The sum of gluino and squark single decay width is expected to be much larger than the annihilation decay width of the squark-gluino system. In this case the bound state decay proceeds through the decay of the constituents and for decay rates of several GeV no well defined bound states exist. Nevertheless, final state interaction will lead to a significant lowering of the effective production threshold, to an enhancement of the cross section and a strong distortion of the differential cross section, in particular of the distribution in the invariant mass of the gluino-squark pair, with details depending on the masses and single decay widths of gluinos and squarks.

This scenario has many similarities with hadronic top quark- 15, 18] and gluino-pair production [19, 20] (with $m_{\tilde{q}}$ close to $m_{\tilde{g}}$ ) close to threshold. (It differs, however, from gluino pair production in the case of large squark masses, where annihilation decays of $(\tilde{g} \tilde{g})$ bound states into two gluon jets could still dominate above constituent decays [21 27].) In particular the distribution in the invariant mass of the gluino-squark pair can be calculated with similar methods. 
A gluino-squark pair can be combined into bound states transforming under three irreducible representations, two of them attractive and one repulsive. As in Refs. [16, 19] the NLO result for the hard corrections is approximated by including the logarithmically enhanced terms from initial- and final-state radiation. The Green's function is evaluated in NLO approximation.

The paper will be organized as follows: For a self-contained treatment we recall in Section2 the quantum numbers of the bound states, discuss various SUSY scenarios and present the qualitative features of threshold production for the case of interest, i.e. for gluinosquark bound states with decay rates comparable to the level spacing of the would-be bound states.

In Section 3.1 we will present the threshold enhancement (or suppression) for the various colour configurations using Green's functions in NLO approximation. These will be evaluated similar to those of the $t \bar{t}$ system discussed in Refs. [16, 17] or the $\tilde{g} \tilde{g}$ system studied in [19,20]. (For earlier investigations of squark and gluino bound state production at hadron colliders see [21 27].) The important difference is the non-degeneracy of the constituent masses, namely the gluino and squark mass. In Section 3.2 the effect of initial- and final-state radiation is investigated. Only $S$ waves will be considered. The choice of the proper value of the strong coupling $\alpha_{s}$ is discussed.

Using this input, the hadronic production cross section can be evaluated in a straightforward way in Section! We limit the discussion to proton-proton collisions at 7 and 14 $\mathrm{TeV}$ and give results for several of the SUSY scenarios discussed in Section2 and compare the results to those obtained without final-state corrections. Section 5 contains our conclusions.

\section{SUSY scenarios, gluino-squark bound states and threshold behaviour}

Let us briefly recall the quantum numbers of gluino-squark pairs in the threshold region, classified according to their colour configurations [27]. While the combination of a spin $1 / 2$ and a spin 0 particle is trivial, a colour-octet and a colour-triplet state can be combined into irreducible representations as follows (See also Appendix A, ):

$$
3 \otimes 8=3 \oplus \overline{\mathbf{6}} \oplus \mathbf{1 5},
$$

while the same equation with a conjugate triplet on the left-hand side (representing an antisquark) results in

$$
\overline{3} \otimes 8=\overline{3} \oplus 6 \oplus \overline{\mathbf{1 5}} .
$$

The interaction can be either attractive or repulsive. In lowest order the coefficient of the QCD potential which governs the final state interaction is given by the expectation value 


\begin{tabular}{c|ccc}
$\mathrm{R}$ & $C_{R}$ & $T^{a, 1} \cdot F^{a, 2}$ & interaction \\
\hline $\mathbf{3}, \overline{\mathbf{3}}$ & $\frac{4}{3}$ & $-\frac{3}{2}$ & attractive \\
$\mathbf{6}, \overline{\mathbf{6}}$ & $\frac{10}{3}$ & $-\frac{1}{2}$ & attractive \\
$\mathbf{1 5}, \overline{\mathbf{1 5}}$ & $\frac{16}{3}$ & $\frac{1}{2}$ & repulsive
\end{tabular}

Table 1: Colour interaction of a $S U(3)$ octet and triplet respectively antitriplet.

of the product of the colour generators of the fundamental and the adjoint representation $T_{i j}^{a} F_{k l}^{a}$. This product, in turn, can be expressed by the eigenvalues of the quadratic Casimir operator of the constituents, $C_{F}=4 / 3$ and $C_{A}=3$, and of the boundstate in representation $R, C_{R}=\left(T^{a}+F^{a}\right)_{R}^{2}$ :

$$
T^{a, 1} \cdot F^{a, 2}=\frac{1}{2}\left[\left(T^{a, 1}+F^{a, 2}\right)_{R}^{2}-\left(T^{a, 1}\right)^{2}-\left(F^{a, 2}\right)^{2}\right]=\frac{1}{2}\left(C_{R}-C_{F}-C_{A}\right) .
$$

The results are listed in Tab. 1 .

The interaction potential between gluino and squark in lowest order is then given by the "Coulomb" potential and depends on the distance $r \equiv|\mathbf{r}|$ between the two constituents

$$
V_{C, \text { Born }}^{[R]}(\mathbf{r})=-\frac{C^{[R]} \alpha_{s}}{r}
$$

with

$$
C^{[R]}=C^{[\bar{R}]}=\frac{3}{2}, \frac{1}{2},-\frac{1}{2} \quad \text { for } \quad R=\mathbf{3}, \mathbf{6}, \mathbf{1 5} .
$$

For the cases with negative (positive) coefficients, corresponding to attraction (repulsion), the cross section will be enhanced (suppressed). NLO corrections are discussed in the next chapter.

The amplitudes for the production and decay of gluino and squark have to be disentangled according to the corresponding representation. It is convenient to define the projectors

$$
\mathbb{P}_{a i, b j}^{[R]} \equiv a^{[R]} \delta_{a b} \mathbb{1}_{i j}+b^{[R]} d_{a b m} T_{i j}^{m}+c^{[R]} i f_{a b m} T_{i j}^{m},
$$

with the coefficients listed in Tab. 2 which obey

$$
\begin{aligned}
\sum_{i} \mathbb{P}_{a j, b k}^{\left[R_{i}\right]} & =\delta_{a b} \mathbb{1}_{j k}, \\
\mathbb{P}_{a i, b j}^{\left[R_{1}\right]} \mathbb{P}_{b j, c k}^{\left[R_{2}\right]} & =\mathbb{P}_{a i, c k}^{\left[R_{1}\right]} \delta_{R_{1} R_{2}}, \\
\mathbb{P}_{a i, a i}^{[R]} & =R .
\end{aligned}
$$

The corresponding projectors for a general $S U(N)$ and a sketch of their derivation are given in Appendix $\left[\mathrm{A}\right.$, together with the results for the coefficient of the potential, $C^{[R]}$. 


\begin{tabular}{c||c|c|c}
$R$ & $a^{[R]}$ & $b^{[R]}$ & $c^{[R]}$ \\
\hline $\mathbf{3}, \overline{\mathbf{3}}$ & $\frac{1}{8}$ & $\frac{3}{8}$ & $\frac{3}{8}$ \\
$\mathbf{6}, \overline{\mathbf{6}}$ & $\frac{1}{4}$ & $-\frac{3}{4}$ & $\frac{1}{4}$ \\
$\mathbf{1 5}, \overline{\mathbf{1 5}}$ & $\frac{5}{8}$ & $\frac{3}{8}$ & $-\frac{5}{8}$
\end{tabular}

Table 2: Coefficients of the projectors from Eq. (6) within $S U(3)$.
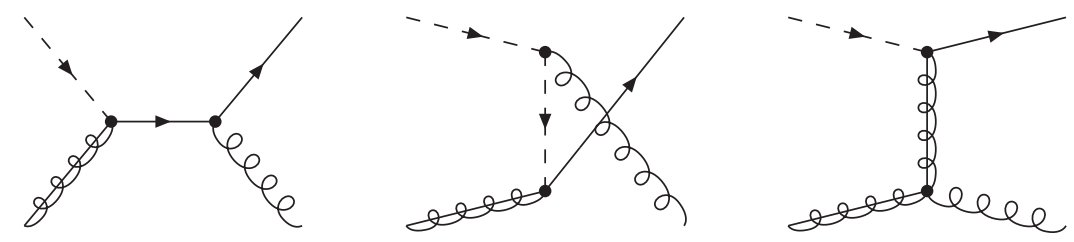

Figure 1: Feynman diagrams contributing at LO to $\tilde{g} \tilde{q} \rightarrow g q$.

Restricting ourselves to the threshold region, only S-wave configurations will be retained. The cross section of states with higher angular momenta is suppressed by at least two powers of the relative velocity $v$ of the two constituents. The classification described in Tab. 1 is applicable to bound state and continuum production and will be important for the description of final state interaction.

As mentioned in the Introduction the phenomenology of bound state production is governed by the relative size of the sum of the decay rates of constituents, i.e. of gluino and squark, $\Gamma_{\tilde{g}}+\Gamma_{\tilde{q}}$, compared to the the level spacing $\Delta M$ between the ground state and the first radial excitation of the strongest bound colour triplet configuration. The choice of $\Delta M$ is motivated by the fact that the binding energy per se depends evidently on the choice of the mass definition (pole mass, potential subtracted mass, ...) while $\Delta M$ is convention independent. The full decay rate of the gluino-squark system is given as sum of the two single decay rates plus the annihilation decay rate.

The annihilation of the gluino-squark system is dominated by the strong decay into a gluon and a quark. The corresponding Feynman diagrams are given in Fig. 1. We assume massless quarks and "Minimal Flavour Violation" (MFV) where squarks couple only the quarks of the same flavour. Let us in a first step evaluate the amplitude for $\tilde{g} \tilde{q}$ production by a gluon and a quark, a result also required for the gluino-squark annihilation. At threshold (i.e. for $p_{\tilde{g}}=m_{\tilde{g}} /\left(m_{\tilde{g}}+m_{\tilde{q}}\right) P$ and $p_{\tilde{q}}=m_{\tilde{q}} /\left(m_{\tilde{g}}+m_{\tilde{q}}\right) P$ ) the amplitude $\mathcal{M}$ can 
be decomposed into the contributions from three irreducible representations

$$
\begin{aligned}
& \mathcal{M}\left(g q \rightarrow \tilde{g} \tilde{q}_{i}\right)=i \sqrt{2} g^{2} \varepsilon_{\mu}\left(p_{g}\right) \bar{u}\left(p_{\tilde{g}}, m_{\tilde{g}}\right) \\
& \times\left\{\frac{1}{s}\left(m_{\tilde{q}_{i}}+m_{\tilde{g}}\right) \gamma^{\mu} T_{j k}^{b} T_{k l}^{a}\right. \\
& +\frac{1}{t-m_{\tilde{g}}^{2}} \gamma^{\mu}\left(m_{\tilde{g}}-\not \phi_{\tilde{q}}\right) i f_{b a c} T_{j l}^{c} \\
& \left.+\frac{2 p_{\tilde{q}_{i}}^{\mu}}{u-m_{\tilde{q}_{i}}^{2}} T_{j k}^{a} T_{k l}^{b}\right\}\left(P_{R} U_{i, R}-P_{L} U_{i, L}\right) u\left(p_{q}, 0\right), \\
& =-i \frac{\sqrt{2} g^{2}}{6\left(m_{\tilde{g}}+m_{\tilde{q}_{i}}\right) m_{\tilde{g}}} \bar{u}\left(p_{\tilde{g}}, m_{\tilde{g}}\right) \notin\left(p_{g}\right)\left(P_{R} U_{i, R}-P_{L} U_{i, L}\right) u\left(p_{q}, 0\right) \\
& \times\left[\left(m_{\tilde{g}}+9 m_{\tilde{q}_{i}}\right) \mathbb{P}_{b j, a l}^{[\mathbf{3}]}+3\left(m_{\tilde{g}}+m_{\tilde{q}_{i}}\right) \mathbb{P}_{b j, a l}^{[\overline{\mathbf{6}}]}-3\left(m_{\tilde{g}}+m_{\tilde{q}_{i}}\right) \mathbb{P}_{b j, a l}^{[\mathbf{1 5}]}\right] .
\end{aligned}
$$

(Here we have used $p_{g} \cdot \varepsilon\left(p_{g}\right)=P \cdot \varepsilon\left(p_{g}\right)=0$.) with the Mandelstam variables $s, t, u$ and the elements of the orthogonal squark-mixing matrix

$$
U=\left(\begin{array}{cc}
U_{L L} & U_{L R} \\
U_{R L} & U_{R R}
\end{array}\right)
$$

The index $i$ in Eqs. (8a), (8b) labels the chiralities $R$ and $L$. For the decay of the triplet and sextet ground state we find (in agreement 1 with [28])

$$
\begin{aligned}
\Gamma\left((\tilde{g} \tilde{q})_{\mathbf{3} / \mathbf{3}} \rightarrow g q\right) & =\frac{\alpha_{s}^{2}\left|R_{1}^{[3]}(0)\right|^{2}\left(m_{\tilde{g}}+9 m_{\tilde{q}}\right)^{2}}{144 m_{\tilde{g}}^{2} m_{\tilde{q}}\left(m_{\tilde{g}}+m_{\tilde{q}}\right)}, \\
\Gamma\left((\tilde{g} \tilde{q})_{\mathbf{6} / \mathbf{6}} \rightarrow g q\right) & =\frac{\alpha_{s}^{2}\left|R_{1}^{[\mathbf{6}]}(0)\right|^{2}\left(m_{\tilde{g}}+m_{\tilde{q}}\right)}{16 m_{\tilde{g}}^{2} m_{\tilde{q}}}
\end{aligned}
$$

The elements of the squark-mixing matrix drop out after summation over quark helicities and in the limit of massless quarks.

The radial part of the Schrödinger wave function at the origin can be derived from the one of the quarkonia or gluinonia by replacing the colour factor and the reduced mass

$$
\left|R_{n}^{[\mathbf{3} / \mathbf{6}]}(0)\right|^{2}=\frac{4\left(C^{[\mathbf{3} / \mathbf{6}]} \alpha_{s}\right)^{3} m_{\mathrm{red}}^{3}}{n^{3}}
$$

with $m_{\text {red }}=m_{\tilde{g}} m_{\tilde{q}} /\left(m_{\tilde{g}}+m_{\tilde{q}}\right)$. Numerically, for $m_{\tilde{g}} \sim m_{\tilde{q}} \sim m \sim 1 \mathrm{TeV}$, this leads to a decay rate $\Gamma=(75 / 32) \alpha_{s}^{2} \alpha_{s}^{3} m$ which is of $\mathcal{O}(10 \mathrm{MeV})$ and thus dramatically smaller than the constituent decay rate discussed below. Gluino-squark annihilation into quarks

\footnotetext{
${ }^{1}$ We thank Y. Kats and D. Kahawala for correspondence on this point.
} 

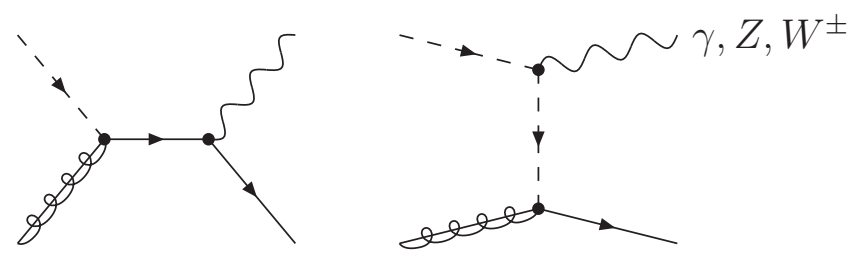

Figure 2: Feynman diagrams contributing at LO to $\tilde{g} \tilde{q} \rightarrow \gamma q, Z q, W^{ \pm} q^{\prime}$.

and electroweak gauge bosons (Fig. 2) is further suppressed by the electromagnetic or weak coupling and thus completely negligible.

The threshold behaviour, i.e. the dependence of the cross section on the invariant mass of the gluino-squark system is governed by the relative size of the constituent decay rate and the binding energy, or more precisely, the excitation energy of the dominant ground state $\Delta M \equiv E_{2}^{[\mathbf{3}]}-E_{1}^{[\mathbf{3}]}$ with

$$
E_{n}^{[\mathbf{3}]}=-\frac{\left(C^{[\mathbf{3}]} \alpha_{s}\right)^{2} m_{\mathrm{red}}}{2 n^{2}}
$$

(Higher order corrections are included in our evaluation of the Green's function in Section 3.1.)

In Tab. 3 the sum of the single decay rates, calculated with the program SDECAY [29], is compared to the level spacing $\Delta M$ for the 17 benchmark points defined in Tab. 4 of Ref. [20] (the spectrum of the SUSY masses has been derived with SuSpect [30]). Due to MFV and the structure of the proton only the gluino-squark systems of the first generation are of interest. For the level spacing $\Delta \bar{M}_{\tilde{g} \tilde{q}}$ the averaged squark mass of the first generation

$$
\bar{m}_{\tilde{q}} \equiv \frac{m_{\tilde{u}_{L}}+m_{\tilde{u}_{R}}+m_{\tilde{d}_{L}}+m_{\tilde{d}_{R}}}{4}
$$

has been used. Obviously the sum of the single decay rates is of order $\Delta \bar{M}_{\tilde{g} \tilde{q}}$ (benchmark points (a)-(i) and (o)-(q)) or far larger (benchmark points (j)-(n)). In the latter cases the width of the gluino is small but the one of the squark is huge in comparison with the mass splitting $\Delta \bar{M}_{\tilde{g} \tilde{q}}$.

The annihilation decay rate is always negligible and thus not shown here. Qualitatively the dominance of constituent decays is easily understood: For squark masses significantly larger than gluino masses the squark decay rate with its kinematically enhanced two-body mode becomes large. In the opposite case the gluino decay rate increases. Only in the degenerate case $m_{\tilde{g}} \sim m_{\tilde{q}}$ both rates are relatively small, i.e. comparable to $\Delta M$, and it is only in this case that resonant structures will arise. Nevertheless, even in the case where the decay rate is significantly larger than $\Delta M$, final state interaction will lead to

\footnotetext{
${ }^{2}$ Final state interaction for $\tilde{g} \tilde{q}$ in the sextet representation is suppressed by powers of $C^{[\mathbf{6}]} / C^{[3]}=1 / 3$.
} 


\begin{tabular}{|c|c|c|c|c|c|c|c|}
\hline $\begin{array}{c}\text { benchmark } \\
\text { point }\end{array}$ & $\begin{array}{c}m_{\tilde{g}} \\
{[\mathrm{GeV}]}\end{array}$ & $\begin{array}{c}\bar{m}_{\tilde{q}} \\
{[\mathrm{GeV}]}\end{array}$ & $\begin{array}{c}\Gamma_{\tilde{g}}+\Gamma_{\tilde{u}_{L}} \\
{[\mathrm{GeV}]}\end{array}$ & $\begin{array}{c}\Gamma_{\tilde{g}}+\Gamma_{\tilde{u}_{R}} \\
{[\mathrm{GeV}]}\end{array}$ & $\begin{array}{c}\Gamma_{\tilde{g}}+\Gamma_{\tilde{d}_{L}} \\
{[\mathrm{GeV}]} \\
\end{array}$ & $\begin{array}{c}\Gamma_{\tilde{g}}+\Gamma_{\tilde{d}_{R}} \\
{[\mathrm{GeV}]} \\
\end{array}$ & $\begin{array}{l}\Delta \bar{M}_{\tilde{g} \tilde{q}} \\
{[\mathrm{GeV}]}\end{array}$ \\
\hline (a) & 606.11 & 555.39 & 10.00 & 5.69 & 9.80 & 4.83 & 3.28 \\
\hline (b) & 493.05 & 453.62 & 7.65 & 4.07 & 7.40 & 3.38 & 2.82 \\
\hline (c) & 381.45 & 349.95 & 6.12 & 3.31 & 5.80 & 2.78 & 2.34 \\
\hline (d) & 717.12 & 655.32 & 12.37 & 7.35 & 12.21 & 6.34 & 3.72 \\
\hline (e) & 826.71 & 753.94 & 14.73 & 9.02 & 14.58 & 7.85 & 4.13 \\
\hline (f) & 935.18 & 851.53 & 17.05 & 10.68 & 16.92 & 9.36 & 4.53 \\
\hline (g) & 1042.60 & 948.11 & 19.33 & 12.31 & 19.22 & 10.84 & 4.91 \\
\hline (h) & 1149.42 & 1044.12 & 21.57 & 13.91 & 21.47 & 12.29 & 5.29 \\
\hline (i) & 936.42 & 859.67 & 16.42 & 9.97 & 16.28 & 8.63 & 4.55 \\
\hline (j) & 802.21 & 1554.00 & 79.95 & 65.25 & 80.04 & 62.71 & 5.16 \\
\hline (k) & 566.65 & 1299.75 & 79.29 & 67.00 & 79.40 & 64.93 & 4.13 \\
\hline (l) & 319.59 & 1055.75 & 79.34 & 69.31 & 79.45 & 67.68 & 2.90 \\
\hline (m) & 1030.98 & 1812.80 & 81.51 & 64.35 & 81.59 & 61.35 & 6.08 \\
\hline (n) & 1255.61 & 2073.80 & 83.80 & 64.20 & 83.87 & 60.71 & 6.95 \\
\hline (o) & 933.03 & 841.02 & 17.63 & 11.36 & 17.50 & 10.06 & 4.50 \\
\hline (p) & 734.11 & 753.47 & 9.59 & 3.34 & 9.52 & 2.17 & 3.95 \\
\hline (q) & 719.66 & 664.46 & 17.96 & 12.85 & 17.87 & 11.82 & 3.74 \\
\hline
\end{tabular}

Table 3: Comparison of the gluino mass, the averaged squark mass of the first generation, the sum of the single decay widths and the level spacing for 26 benchmark points defined in Tab. 4 of Ref. [20]. 
a broad enhancement. Therefore results for two benchmark points will be presented in Section 4 , one with $\Gamma_{\tilde{g}}+\Gamma_{\tilde{q}}$ comparable to $\Delta M$, the second one with $\Gamma_{\tilde{g}}+\Gamma_{\tilde{q}}$ significantly larger.

Hence formation of bound states [26] or of sharp resonances in the differential cross section (as discussed for the gluino-pair production in [19,20]) is not possible here. Nevertheless, for the benchmark points (a)-(i) and (o)-(q) the binding effects of the colour triplet and octet will be visible in the differential cross section.

The technical aspects of the calculation for threshold production are very similar to the ones for top-quarks [16, 17] and gluinos [19, 20]. The cross section, differential in $M$, the invariant mass of the gluino-squark pair, can be decomposed into a factor representing the hard, short distance part and a factor determined by the imaginary part of the Green's function, evaluated at the origin. This partonic cross section is then convoluted with the luminosity function:

$$
M \frac{\mathrm{d} \sigma_{P P \rightarrow R}}{\mathrm{~d} M}\left(S, M^{2}\right)=\sum_{i, j} \int_{\rho}^{1} \mathrm{~d} \tau\left[\frac{\mathrm{d} \mathcal{L}_{i j}}{\mathrm{~d} \tau}\right]\left(\tau, \mu_{F}^{2}\right) M \frac{\mathrm{d} \hat{\sigma}_{i j \rightarrow R}}{\mathrm{~d} M}\left(\hat{s}, M^{2}, \mu_{R}^{2}, \mu_{F}^{2}\right),
$$

with

$$
\begin{aligned}
M \frac{\mathrm{d} \hat{\sigma}_{i j \rightarrow R}}{\mathrm{~d} M}\left(\hat{s}, M^{2}, \mu_{R}^{2}, \mu_{F}^{2}\right) & =\mathcal{F}_{i j \rightarrow R}\left(\hat{s}, M^{2}, \mu_{R}^{2}, \mu_{F}^{2}\right) \frac{4 \operatorname{Im}\left\{G^{[R]}\left(\mathbf{0}, M+\frac{i}{2}\left(\Gamma_{\tilde{g}}+\Gamma_{\tilde{q}_{i}}\right)\right)\right\}}{m_{\mathrm{red}}^{2}} \\
{\left[\frac{\mathrm{d} \mathcal{L}_{i j}}{\mathrm{~d} \tau}\right]\left(\tau, \mu_{F}^{2}\right) } & =\int_{0}^{1} \mathrm{~d} x_{1} \int_{0}^{1} \mathrm{~d} x_{2} f_{i \mid P}\left(x_{1}, \mu_{F}^{2}\right) f_{j \mid P}\left(x_{2}, \mu_{F}^{2}\right) \delta\left(\tau-x_{1} x_{2}\right) .
\end{aligned}
$$

The quantities $S$ and $\hat{s}$ denote as usual the hadronic and the partonic center of mass energy squared, and $\tau \equiv \hat{s} / S$. The lower limit of the integration is given by $\rho \equiv M^{2} / S$. The superscript of the Green's function refers to the colour representation $R$ of the corresponding $S$ wave and $\mu_{F}$ and $\mu_{R}$ denote the factorization and renormalization scale, respectively.

The only reaction present at leading order (LO) is $g q \rightarrow \tilde{g} \tilde{q}_{i}$ where in the Feynman diagrams of Fig. 1 initial and final states have to be exchanged. For the four squark types under consideration and including both squark and antisquark the hard parts of the cross sections are given as

$$
\mathcal{F}_{i j \rightarrow R}^{(0)}=\mathcal{N}_{i j}^{[R]} \frac{\pi^{2} \alpha_{s}^{2}\left(\mu_{R}\right)}{12 \hat{s}} \delta(1-z)
$$

with $z=M^{2} / \hat{s}$. The non-vanishing normalization factors $\mathcal{N}_{i j}^{[R]}$ are obtained from Eqs. (8aa) 
and $(8 \mathrm{bb})$ :

$$
\begin{aligned}
\mathcal{N}_{g q}^{[3]} & =\mathcal{N}_{g \bar{q}}^{[\overline{3}]}=\frac{\left(m_{\tilde{g}}+9 \bar{m}_{\tilde{q}}\right)^{2}}{3 m_{\tilde{g}}\left(m_{\tilde{g}}+\bar{m}_{\tilde{q}}\right)}, \\
\mathcal{N}_{g q}^{[6]} & =\mathcal{N}_{g \bar{q}}^{[6]}=\frac{6\left(m_{\tilde{g}}+\bar{m}_{\tilde{q}}\right)}{m_{\tilde{g}}}, \\
\mathcal{N}_{g q}^{[15]} & =\mathcal{N}_{g \bar{q}}^{[\overline{15}]}=\frac{15\left(m_{\tilde{g}}+\bar{m}_{\tilde{q}}\right)}{m_{\tilde{g}}},
\end{aligned}
$$

which do not depend on the squark mixing.

The Green's function depends on the energy $E=M-\left(m_{\tilde{g}}+m_{\tilde{q}_{i}}\right)$ and on the sum of the decay rates. It is obtained from the non-relativistic Schrödinger equation

$$
\left\{\left[\frac{2(-i \nabla)^{2}}{m_{\mathrm{red}}}+V^{[R]}(\mathbf{r})\right]-\left(E+\frac{i}{2}\left(\Gamma_{\tilde{g}}+\Gamma_{\tilde{q}_{i}}\right)\right)\right\} G^{[R]}\left(\mathbf{r}, M+\frac{i}{2}\left(\Gamma_{\tilde{g}}+\Gamma_{\tilde{q}_{i}}\right)\right)=\delta^{(3)}(\mathbf{r}),
$$

with the potential being $V_{C \cdot \text { Born }}^{[R]}(\mathbf{r})$, the Coulomb potential of Eqs. (44) and (5). NLO corrections to both, the hard parts of the cross section as far as the Green's function are studied in the next section.

\section{$3 \quad$ Next to leading order corrections}

The NLO corrections of the cross section can be split up into those for the imaginary part of the Green's function $\operatorname{Im}\left\{G^{[R]}\right\}$ and those for the hard coefficients $\mathcal{F}_{i j \rightarrow R}$ as defined in Eq. (15).

\subsection{Green's function}

Following the idea of the Green's function method developed in Refs. [31, 32] we start with the interaction potential in position space. With the colour coefficients of Eq. (5) it is given up to NLO

$$
V_{C}^{[R]}(\mathbf{r})=-C^{[R]} \frac{\alpha_{s}\left(\mu_{G}^{[R]}\right)}{r}\left\{1+\frac{\alpha_{s}\left(\mu_{G}^{[R]}\right)}{4 \pi}\left[2 \beta_{0}\left(\ln \left(\mu_{G}^{[R]} r\right)+\gamma_{E}\right)+a_{1}\right]\right\},
$$

The coefficient $a_{1}=31 / 9 C_{A}-20 / 9 T_{F} n_{f}$ is identical to the one relevant for the NLO corrections to the potentials of gluinonia and quarkonia [33]. The coefficient of the QCDbeta function is $\beta_{0}=11 / 3 C_{A}-4 / 3 T_{F} n_{f}$ with $C_{A}=3$ and $T_{F}=1 / 2$ and $\gamma_{E}=0.5772$. The choice of $n_{f}=5$ active quark flavours is motivated by the scale of the Green's function 
(see Eq. (25) ) for the gluino and squark masses under consideration. Effects of massive top quarks could be incorporated, however, are irrelevant at the present level of precision. For both LO and NLO we adopt $\alpha_{s}\left(M_{Z}=91.1876 \mathrm{GeV}\right)=0.12018$ as provided from MSTW2008NLO [34] and employ the two-loop running as provided by RunDec [35].

The Green's function for the top-antitop system is known in compact analytic form [36] (see also [37]) and the result is easily applied to the present case

$$
\begin{aligned}
G^{[R]}\left(E+\frac{i}{2}\left(\Gamma_{\tilde{g}}+\Gamma_{\tilde{q}_{i}}\right)\right) & \equiv G^{[R]}\left(\mathbf{0}, E+m_{\tilde{g}}+m_{\tilde{q}_{i}}+\frac{i}{2}\left(\Gamma_{\tilde{g}}+\Gamma_{\tilde{q}_{i}}\right)\right) \\
& =i \frac{v m_{\mathrm{red}}^{2}}{\pi}+\frac{C^{[R]} \alpha_{s}\left(\mu_{G}^{[R]}\right) m_{\mathrm{red}}^{2}}{\pi}\left[g_{\mathrm{LO}}+\frac{\alpha_{s}\left(\mu_{G}^{[R]}\right)}{4 \pi} g_{\mathrm{NLO}}+\ldots\right],
\end{aligned}
$$

with

$$
\begin{aligned}
g_{\mathrm{LO}} \equiv & L-\psi^{(0)} \\
g_{\mathrm{NLO}} \equiv \beta_{0}\left[L^{2}-2 L\left(\psi^{(0)}-\kappa \psi^{(1)}\right)+\kappa \psi^{(2)}+\left(\psi^{(0)}\right)^{2}-3 \psi^{(1)}-2 \kappa \psi^{(0)} \psi^{(1)}\right. & \\
& \left.\quad+4{ }_{4} F_{3}(1,1,1,1 ; 2,2,1-\kappa ; 1)\right]+a_{1}\left[L-\psi^{(0)}+\kappa \psi^{(1)}\right]
\end{aligned}
$$

and

$$
\kappa \equiv i \frac{C^{[R]} \alpha_{s}\left(\mu_{G}^{[R]}\right)}{2 v}, \quad v \equiv \sqrt{\frac{E+\frac{i}{2}\left(\Gamma_{\tilde{g}}+\Gamma_{\tilde{q}_{i}}\right)}{2 m_{\mathrm{red}}}}, \quad L \equiv \ln \frac{i \mu_{G}^{[R]}}{4 m_{\mathrm{red}} v} .
$$

The $n$-th derivative $\psi^{(n)}=\psi^{(n)}(1-\kappa)$ of the digamma function $\psi(z)=\gamma_{E}+(d / d z) \ln \Gamma(z)$ is evaluated at $(1-\kappa)$. The numerical solution of the Schrödinger equation of Eq. (18) involves multiple poles in the binding energy which have to be resummed to single poles [36]. The definition of the Generalized Hypergeometric Function ${ }_{4} F_{3}$ together with comments about its numerical evaluation can be found in Ref. [26]. In particular, attention has to be paid to the evaluation of ${ }_{4} F_{3}$ at the beginning of a branch cut for its last argument.

\subsection{Short distance corrections}

The complete NLO corrections to the hard part of the cross section involve virtual corrections to the process $g q \rightarrow \tilde{g} \tilde{q}$ plus real radiation (for gluino-pair production see [20]). The production of the final state $\tilde{g} \tilde{u}_{1}$ in colour representation $R$, for example, will receive contributions from the processes $g g \rightarrow R \bar{u}, u g \rightarrow R g, u u \rightarrow R u, u \bar{u} \rightarrow R \bar{u}, u q \rightarrow R q$, $u \bar{q} \rightarrow R \bar{q}$ and $q \bar{q} \rightarrow R \bar{q}$ with $q \neq u$.

For the present analysis the complete calculation has been replaced by an approximation employed in [16, 19] for $t \bar{t}$ and $\tilde{g} \tilde{g}$ production which includes the leading logarithms from 
initial and final state radiation, affecting the subprocess $g q \rightarrow \tilde{g} \tilde{q}$ which was present at LO already. The quality of this approximation can be judged e. g. from the corresponding analysis for top production ( [17, Eq. (14)) where both partial and complete results are available. In this case the size of the corrections depends on the subprocess. For gluon induced processes the corrections are large $(35 \%-40 \%$ of the corrected result) with the enhanced term amounting to about $3 / 4$ of the corrections. For quark induced processes, however, the corrections amount to $25 \%-30 \%$ with roughly equal share of enhanced and non-enhanced terms. One gets

$$
\begin{aligned}
\mathcal{F}_{i j \rightarrow R}= & \mathcal{N}_{i j}^{[R]} \frac{\pi^{2} \alpha_{s}^{2}\left(\mu_{R}\right)}{12 \hat{s}}\left[1+\frac{\alpha_{s}\left(\mu_{R}\right)}{\pi} \frac{\beta_{0}^{\mathrm{SQCD}}}{2} \ln \left(\frac{\mu_{R}^{2}}{\left(m_{\tilde{g}}+\bar{m}_{\tilde{q}}\right)^{2}}\right)\right] \\
& \times\left(\delta(1-z)+\frac{\alpha_{s}\left(\mu_{R}\right)}{\pi} \mathcal{R}^{[R]}(z)\right)
\end{aligned}
$$

with

$$
\begin{aligned}
\mathcal{R}^{[R]}(z)= & \left(C_{A}+C_{F}\right)\left\{2\left[\frac{\ln (1-z)}{1-z}\right]_{+}-\ln \left(\frac{\mu_{F}^{2}}{\left(m_{\tilde{g}}+\bar{m}_{\tilde{q}}\right)^{2}}\right)\left[\frac{1}{1-z}\right]_{+}\right\} \\
& -C_{R}\left[\frac{1}{1-z}\right]_{+}-\delta(1-z) \frac{\beta_{0}^{\mathrm{SQCD}}+3 C_{F}}{2} \ln \left(\frac{\mu_{F}^{2}}{\left(m_{\tilde{g}}+\bar{m}_{\tilde{q}}\right)^{2}}\right),
\end{aligned}
$$

where $\beta_{0}^{\mathrm{SQCD}}=3 C_{A}-2 T_{F} n_{f}$ is the one-loop coefficient of the SUSY QCD beta function. The normalization factors $\mathcal{N}_{i, j}^{[R]}$ are given in Eq. (17), the constants $C_{R}$ in Tab. 1. The conventional plus-distribution 3 has been employed to regularize singularities appearing at $z=1$. The terms of the first line of Eq. (24) stem from initial-state radiation and are therefore proportinal to the sum of the Casimirs of the adjoint and the fundamental representation. The first term in the second line originates from final-state radiation and depends on the representation of the gluino-squark state. The last term originates from the renormalization of the parton densities. We do not include the estimate of the virtual corrections based on the colour summed open production - as performed in [19] for the gluino-gluino case.

\section{Numerical results}

\subsection{Benchmark Points}

From now on we limit our discussion to the benchmark points (p) and (q). First we focus on (p), where the gluino mass and the four squark masses of the first generation lie in

\footnotetext{
${ }^{3}$ The plus-distribution follows the prescription $\int_{0}^{1} d z\left[\frac{\ln ^{n}(1-z)}{1-z}\right]_{+} f(z) \equiv \int_{0}^{1} d z \frac{\ln ^{n}(1-z)}{1-z}[f(z)-f(1)]$ for $n=0,1, \ldots$ and any test function $f(z)$. If the lower integration boundary is given by $\rho>0$ the plus distribution can be replaced by the $\rho$-description via $\left[\frac{\ln ^{n}(1-z)}{1-z}\right]_{+} \rightarrow \frac{\ln ^{n+1}(1-\rho)}{n+1} \delta(1-z)+\left[\frac{\ln ^{n}(1-z)}{1-z}\right]_{\rho}$ where the latter is defined through $\int_{\rho}^{1} d z\left[\frac{\ln ^{n}(1-z)}{1-z}\right]_{\rho} f(z) \equiv \int_{\rho}^{1} d z \frac{\ln ^{n}(1-z)}{1-z}[f(z)-f(1)]$.
} 
a range of less than $30 \mathrm{GeV}$. For the right handed squarks the sum of the decay widths is slightly smaller than the level spacing $\Delta \bar{M}_{\tilde{g} \tilde{q}}$, for the left handed ones approximately twice as big. Thus, the enhancement from the lowest lying resonance will be visible in the differential cross section.

As an alternative we consider benchmark point (q). In this case the constituent decay rates are significantly larger than the level spacing. Final state interaction will, nevertheless, lead to a significant enhancement and distortion of the cross section in the threshold region.

\subsection{Green's Function}

As a characteristic scale for the strong coupling in the potential we have chosen the Bohr radius

$$
\mu_{G}^{[R]} \equiv 2 m_{\text {red }}\left|C^{[R]}\right| \alpha_{s}\left(\mu_{G}^{[R]}\right)
$$

The normalized imaginary part of the Green's function at the origin is displayed in Fig. 3. The plots involving the thresholds with up-type (down-type) squarks can be found at the top (at the bottom) while left-handed (right-handed) squarks can be found on the left (right) side. LO and NLO curves are displayed separately and the poor convergence of the binding energies, which had been observed for quarks [38 40] as well as for gluinos [26], results in a shift of $2 \mathrm{GeV}$ between the ground state of the most attractive colour-triplet configuration going from LO to NLO. The effect of the larger decay widths for the lefthanded squarks manifests itself in the smearing of this resonance compared to the righthanded particles. The attractive potential of the colour-sextet representation is suppressed by a factor of three such that no resonances can be seen. The contribution of the repulsive 15 configuration is negligible in the threshold region and the LO and NLO curve lie on top of each other.

\subsection{Cross section}

Combining the results of the previous section, the threshold behaviour of gluino-squark production in proton-proton collision can be computed. For the numerical evaluation of the strong coupling we used the program RunDec [35] and the starting values $\alpha_{s}\left(M_{Z}\right)=$ 0.13939 for the MSTW2008LO PDFs and $\alpha_{s}\left(M_{Z}\right)=0.12018$ for the MSTW2008NLO set [34. For $\mu_{H}$ (Eq. (26) ) the running is performed at the two-loop level. Decoupling of the top-quark at $m_{t}=172.0 \mathrm{GeV}$ and decoupling of the gluino and squarks at $\mu_{H}$ is performed at one-loop precision (see for example [41]).

For simplicity the squark mass entering the reduced mass of the cross section and the determination of the coupling is understood as averaged mass of the squarks of the first generation from Eq. (13). The information of the four different squarks is only encoded 

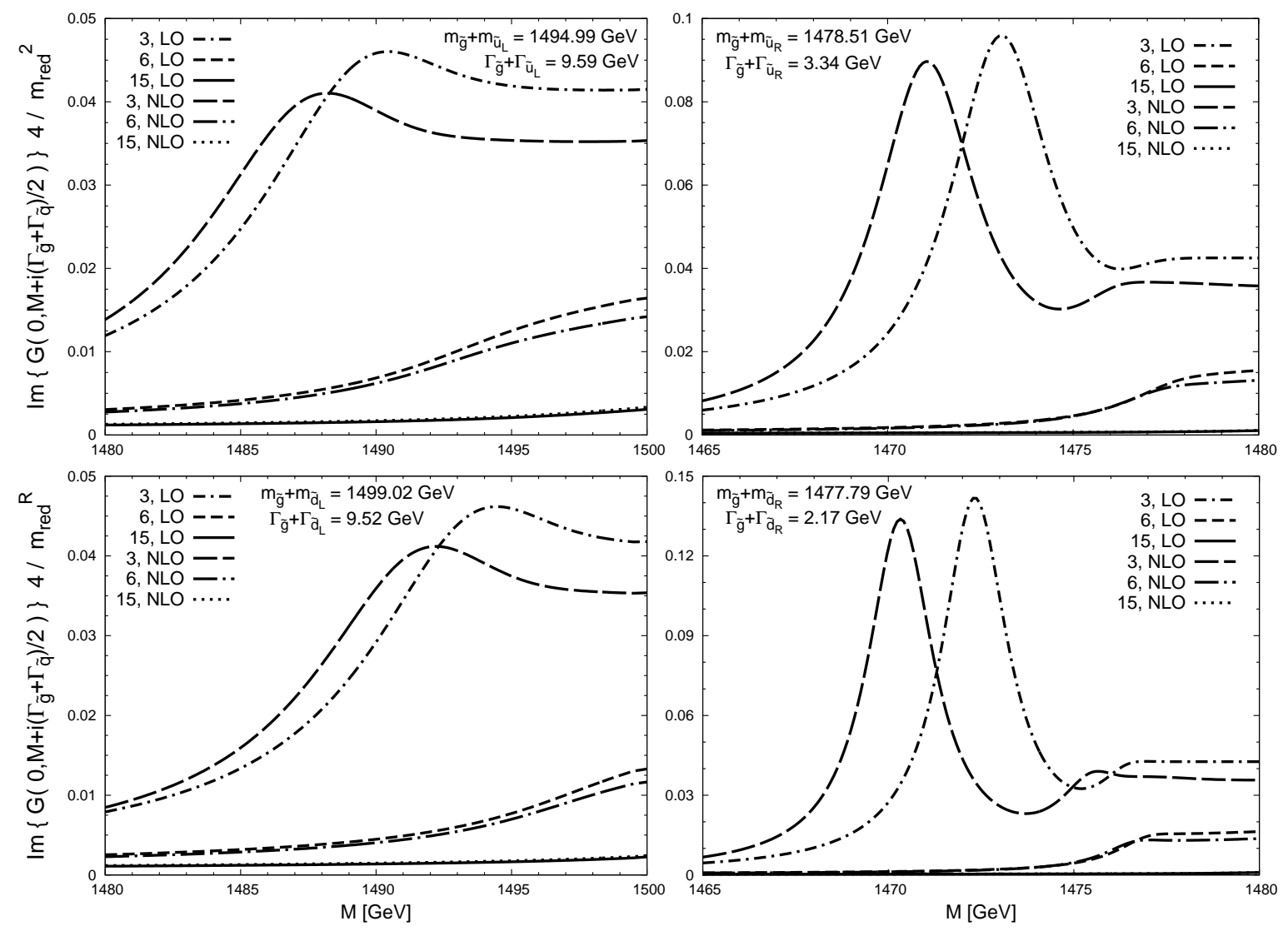

Figure 3: Imaginary part of the Green's function for scenario (p) for the up-type (at the top) and the down-type (at the bottom) squarks. LO and NLO curves are plotted for all three colour configurations, whereas both curves lie on top of each other for the $\mathbf{1 5}$ representation.

in the decay widths $\Gamma_{\tilde{q}_{i}}$ and in the total mass of the gluino-squark system entering the Green's function via the relative velocity $v$ from Eq. (22) and determining the position of the threshold.

In Fig. 4 the LO result for the production cross section, differential in the invariant mass of the two supersymmetric particles, can be found. The contributions of the channels $g q \rightarrow \tilde{g} \tilde{q}$ and $g \bar{q} \rightarrow \tilde{g} \tilde{q}^{*}$ are both included. We have employed the parton distribution functions (PDF) of MSTW2008LO [34]. Both factorization and the renormalization scale have been set to the hard scale

$$
\mu_{F}=\mu_{R}=\mu_{H} \equiv m_{\tilde{g}}+\bar{m}_{\tilde{q}}
$$

Up to NLO Green's function and hard kernel are separately renormalization scale invariant, such that the cross section involves the two scales $\mu_{G}^{[R]}$ and $\mu_{H}$.

The upper four plots of Fig. 4 are arranged as in Fig. 3. They show the contributions 

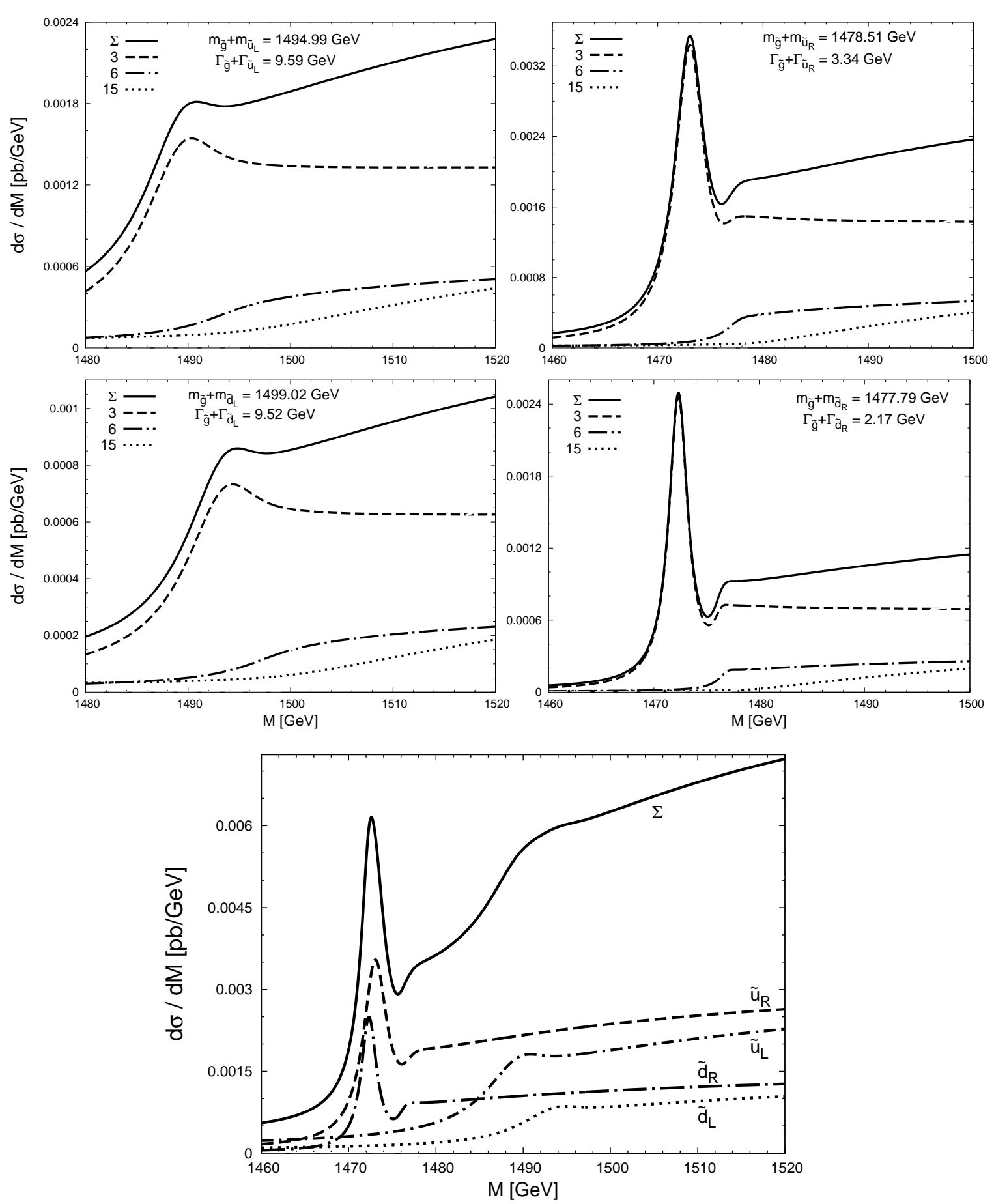

Figure 4: Prediction for the invariant mass distribution for scenario (p) at LO. The upper four plots represent the results for the configurations separately and are ordered as in Fig. 3. The figure at the bottom displays the individual contributions from $\tilde{u}_{L, R}$ and $\tilde{d}_{L, R}$ and their sum. 
from the three representations and their sum. In the lower plot the contributions of the four gluino-squark channels are shown as well as their sum. It is clearly visible that, due to MFV and the quark content of the proton, the contributions from up-type squarks are twice as big as the ones of the curves involving down-type squarks. The resonances of the right-handed squarks nearly coincide so that a relatively sharp resonance will be visible in the invariant mass distribution.

For degenerated squark and gluino masses, as it is roughly realized in this scenario (p), the normalization factors of Eq. (17) are comparable for all three colour representations. Therefore, the relative magnitudes of the cross sections are largely governed by the Green's function. Furthermore, the hard kernel does not vary strongly in in the threshold region, hence the shapes of the curves shown in Figs. 3 and 4 exhibit a similar behaviour.

Let us now consider the (approximate) NLO result. The NLO values for the Green's function will be used together with the approximation of Eqs. (23) and (24) for the hard part of the invariant mass distribution.

The result for benchmark point (p) is displayed in Fig. 5 for two regions of the invariant mass. The shapes of LO (Fig. (4) and NLO predictions (Fig. (5) look quite similar, differing mainly in their relative normalization by about $20 \%$. Furthermore, the difference between the masses of $\tilde{u}_{L}$ and $\tilde{u}_{R}$, respectively, becomes irrelevant for high energies, hence the difference between the configurations with left- and righthanded squarks vanishes far above the threshold. The contribution of the $\tilde{g} \tilde{u}_{L / R}$ final states is twice the contribution of the down-type squarks due to the structure of the proton. We also show the sum of the fixed order (FO) curves where the Green's function is replaced by its leading perturbative expansion in $\alpha_{s}$, furthermore, in the limit $\Gamma_{\tilde{g}}, \Gamma_{\tilde{q}_{i}} \rightarrow 0$. Hence one gets

$$
\operatorname{Im}\left\{G^{[R]}\left(M+\frac{i}{2}\left(\Gamma_{\tilde{g}}+\Gamma_{\tilde{q}_{i}}\right)\right)\right\} \rightarrow \frac{m_{\mathrm{red}}^{2}}{\pi} v\left(1+C^{[R]} \frac{\alpha_{s} \pi}{2 v}\right) .
$$

with $v$ as in Eq. (22). In the threshold region the four different values of the sum $m_{\tilde{g}}+m_{\tilde{q}_{i}}$ are clearly visible from the kinks of the dashed FO curve. Above about $M=1700 \mathrm{GeV}$ the two curves of the FO ansatz and the one using the full Green's function roughly coincide and binding effects are no longer of importance. The integrated difference between the two functions

$$
\Delta \sigma \equiv \int_{M_{0}}^{M_{1}} \mathrm{~d} M\left[\left(\frac{\mathrm{d} \sigma}{\mathrm{d} M}\right)_{\text {Green }}-\left(\frac{\mathrm{d} \sigma}{\mathrm{d} M}\right)_{\mathrm{FO}}\right],
$$

for $M_{0}=1415 \mathrm{GeV}$ and $M_{1}=1650 \mathrm{GeV}$ represents a measure for the effect of the final state interaction and amounts to a relative enhancement of about $2.57 \%$ compared to the total cross section of $\sigma_{\text {tot }}=7.29 \mathrm{pb}$ which has been calculated at NLO using Prospino2 [5]. It should be mentioned that also effects of squarks from the second and third generation are included in the total cross section.

The sensitivity of our results on the factorization scale $\mu_{F}$ and the renormalization scale $\mu_{R}$ is shown in Fig. 6, both for LO and NLO predictions. The scales $\mu_{F}$ and $\mu_{R}$ are 

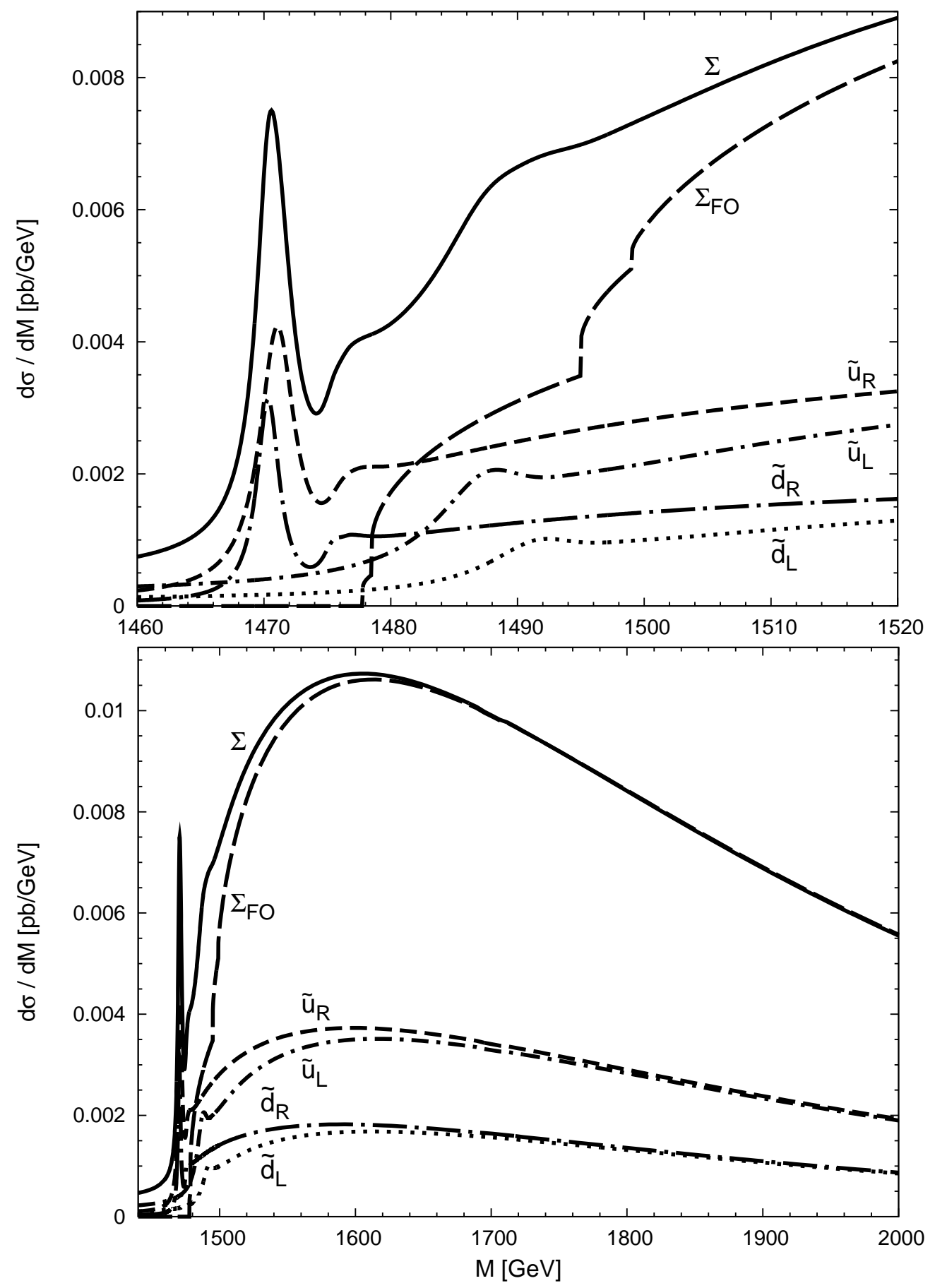

Figure 5: Prediction for differential cross section as function of the invariant mass for scenario (p) evaluated in NLO approximation as defined in Eqs. (23) and (24) for the four squark species. Also shown is the sum of the four squarks (solid curve) and the FO prediction (long dashed curve). 

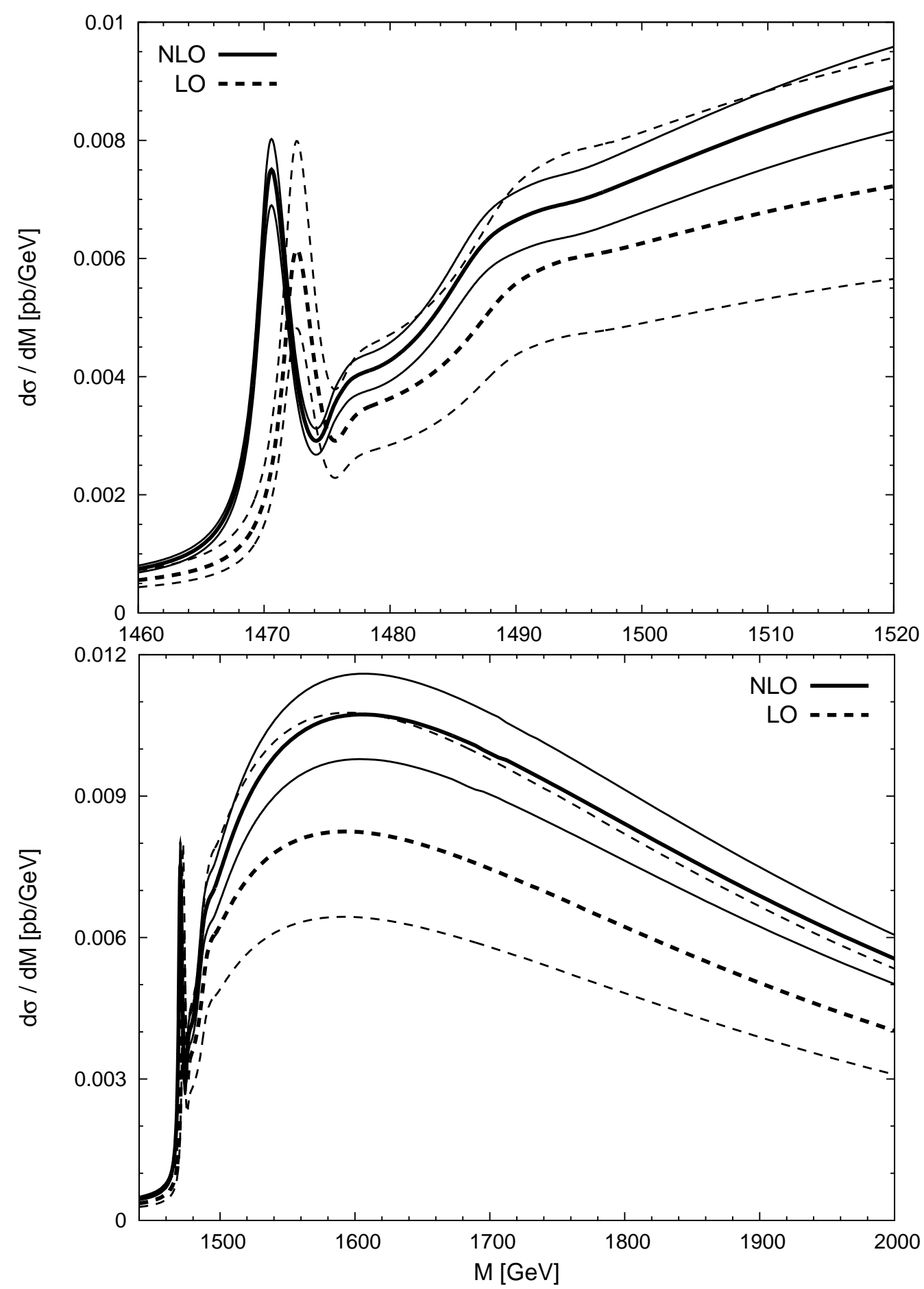

Figure 6: Dependence of the invariant mass distribution on the choice of factorization and renormalization scale for scenario (p). For the scale of the Green's function Eq. (25) has been adopted throughout. 

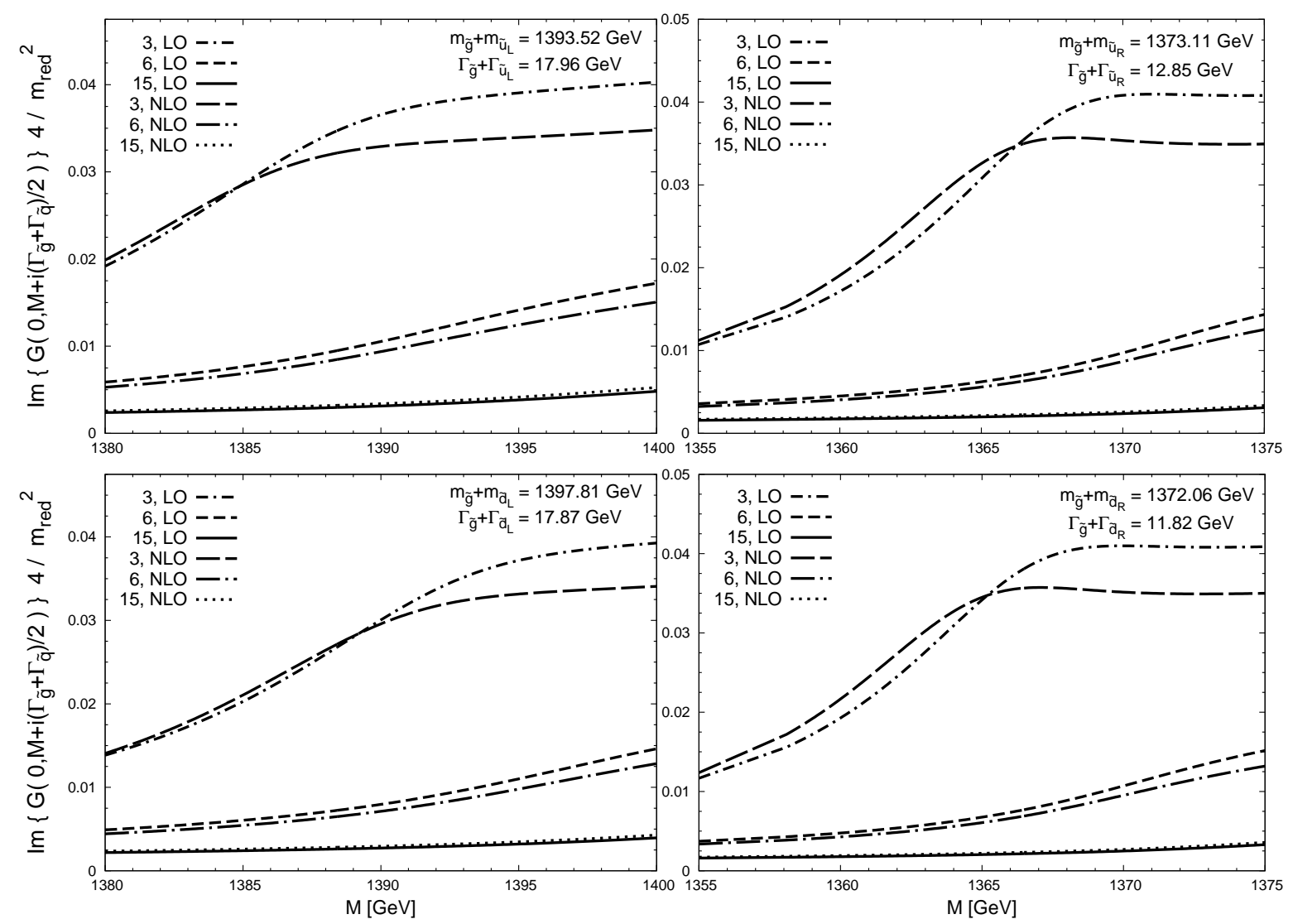

Figure 7: Imaginary part of the Green's function for scenario (q) for the up-type (at the top) and the down-type (at the bottom) squarks. LO and NLO curves are plotted for all three colour configurations, whereas both curves lie on top of each other for the $\mathbf{1 5}$ representation.

chosen to be equal and varied between $\mu_{H} / 2$ and $2 \mu_{H}$ (see Eq. 26). As expected, the scale dependence is significantly reduced for the NLO approximation, amounting to less than $\pm 10 \%$. An estimate of the theoretical uncertainty may either be based on this scale dependence, or, more conservatively, on the difference between LO and NLO results, amounting to roughly $20 \%$.

As a second option we study the prediction for benchmark point (q) with constituent decay rates significantly larger than the level spacing. The Green's functions are shown in Fig. 7. The strong enhancement of the triplet Green's function is evident, as well as the strong suppression for the $\mathbf{1 5}$ representation. The predictions for the differential cross sections are shown in Fig. 8, again separately for the different squark species and the different representation. The step in $\mathrm{d} \sigma / \mathrm{d} M$ slightly below the nominal threshold is still visible for the individual squark species, it is, however, smeared out when one considers the sum of all four channels. Nevertheless, again one observes a significant enhancement 

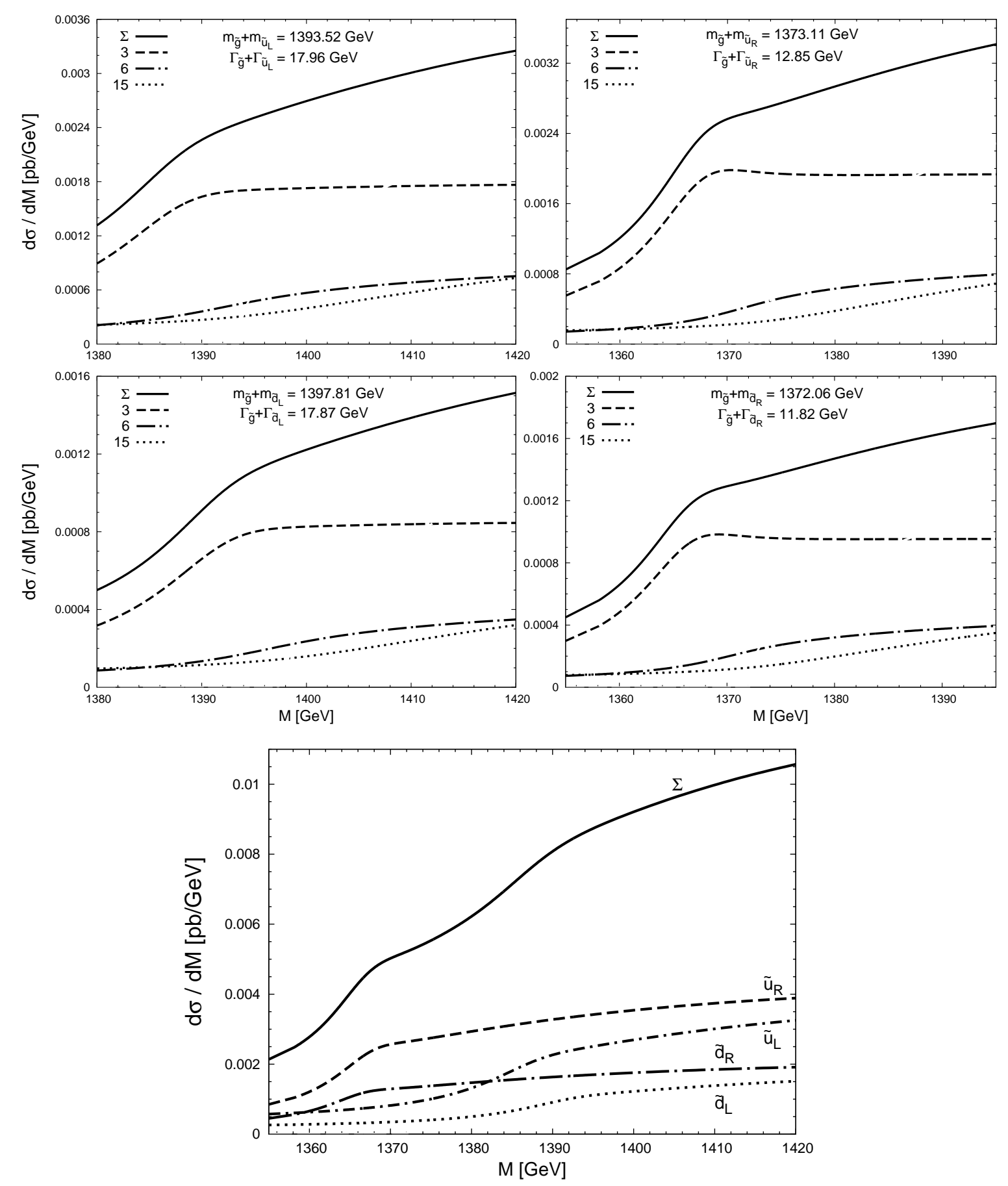

Figure 8: Prediction for the invariant mass distribution for scenario (q) at LO. The upper four plots represent the results for the configurations separately and are ordered as in Fig. 3. The figure at the bottom displays the individual contributions from $\tilde{u}_{L, R}$ and $\tilde{d}_{L, R}$ and their sum. Furthermore, the formalism of the Green's function is compared to the FO method. 
of the differential cross section in the threshold region, which extends from approximately $20 \mathrm{GeV}$ below to $50 \mathrm{GeV}$ above the nominal threshold.

The NLO result for benchmark point (q) is displayed in Fig. 9 again for two regions of the invariant mass. The effect of the NLO correction and the dependence on factorization and renormalization scale is similar to the one discussed for benchmark point $(\mathrm{p})$.

\section{Conclusions}

The threshold behaviour of gluino-squark production at hadron colliders has been investigated. The NLO Green's function has been evaluated for gluino and squark in the three possible irreducible representations with dimensions 3, 6 and 15. The logarithmically enhanced part of the NLO corrections to the "hard kernel" for the evaluation of the cross section is included in the analysis.

In contrast to the case of gluino pairs the constituent decay rate is generically comparable or larger than the binding energy, independent of the choice of the the SUSY parameters. Hence it is only for the exceptional case of gluino and squark masses being comparable, where both gluino and squark decay rates are suppressed, that pronounced resonance behaviour might occur. Nevertheless, attractive final state interaction, in particular for squark and gluino in the triplet representation, will lead to a modification of the cross section in the threshold region. The effective threshold is lowered by about $10 \mathrm{GeV}$ from final state interaction and additional smearing due to the constituent decay rate. Most of the enhancement is concentrated in an interval of about $50 \mathrm{GeV}$ around the nominal location of the threshold. Final state interaction, thus, leads to a significant distortion of the differential distribution $\mathrm{d} \sigma / \mathrm{d} M$. Compared to the total cross section evaluated in NLO without inclusion of the rescattering effects final state interaction increases the result by about $2-3 \%$.

\section{A Tensor product in a general $\mathrm{SU}(\mathrm{N})$}

The tensor product of the adjoint and the fundamental representations of $S U(N)$ can be obtained following standard methods and is given by

$$
\left(N^{2}-1\right) \otimes N=\frac{N(N+2)(N-1)}{2} \oplus \frac{N(N+1)(N-2)}{2} \oplus N
$$

where the second representation on the right hand side of Eq. (29) is understood to be a conjugate one. (See also [27].)

The projectors for these three representations are given as (for $S U(3)$ see also Eq. (6))

$$
\mathbb{P}_{a i, b j}^{[R]}=a^{[R]} \delta_{a b} \mathbb{1}_{i j}+b^{[R]} d_{a b m} T_{i j}^{m}+c^{[R]} i f_{a b m} T_{i j}^{m}
$$



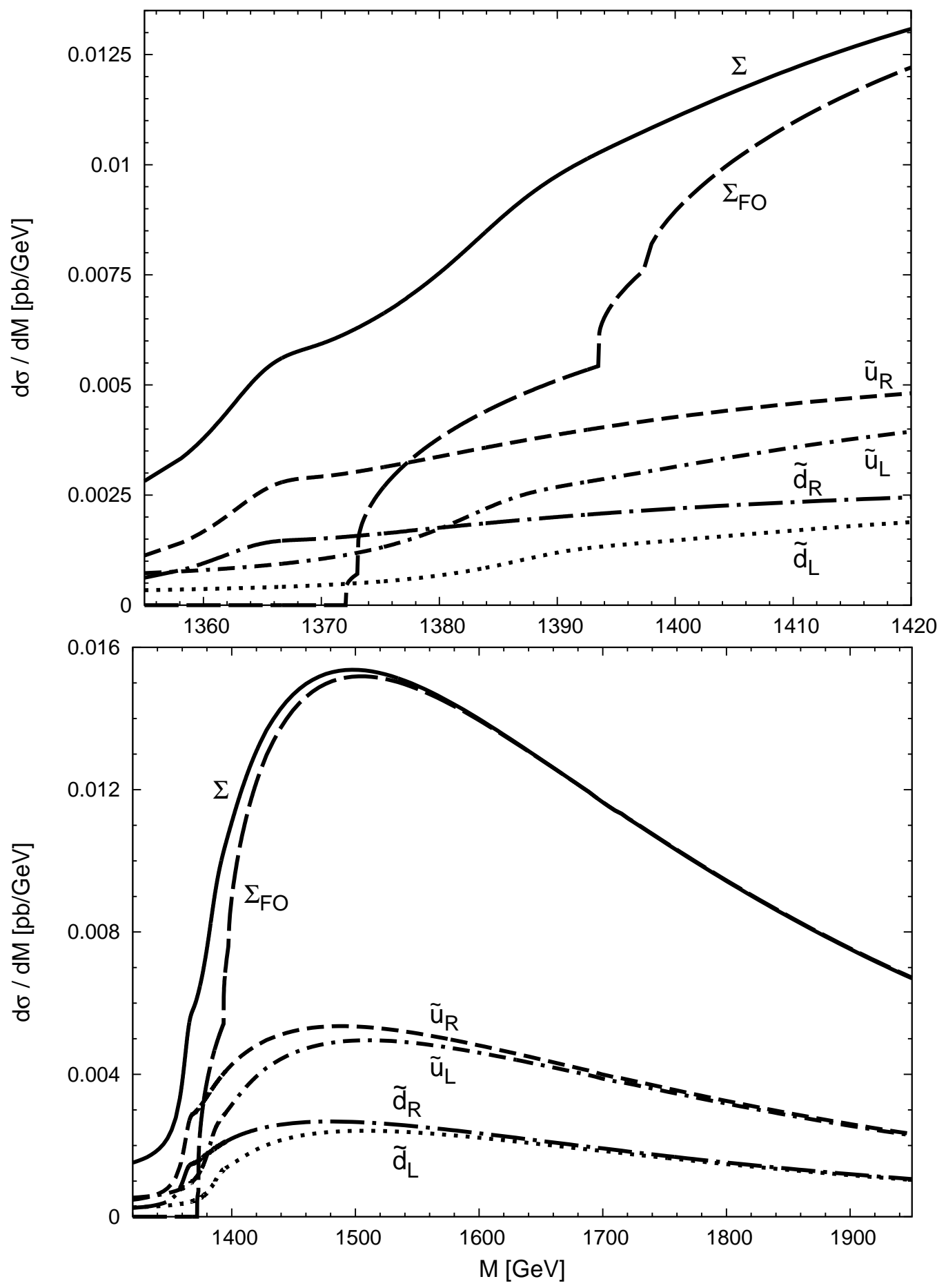

Figure 9: Prediction for differential cross section as function of the invariant mass for scenario (q). The NLO approximation of Eqs. (23) and (24) has been adopted. 


\begin{tabular}{c||c|c|c||c}
$R$ & $a^{[R]}$ & $b^{[R]}$ & $c^{[R]}$ & $C^{[R]}$ \\
\hline \hline$N$ & $\frac{1}{N^{2}-1}$ & $\frac{N}{N^{2}-1}$ & $\frac{N}{N^{2}-1}$ & $\frac{N}{2}$ \\
\hline$\frac{N(N+1)(N-2)}{2}$ & $\frac{N-2}{2(N-1)}$ & $-\frac{N}{2(N-1)}$ & $\frac{N-2}{2(N-1)}$ & $\frac{1}{2}$ \\
\hline$\frac{N(N+2)(N-1)}{2}$ & $\frac{N+2}{2(N+1)}$ & $\frac{N}{2(N+1)}$ & $-\frac{N+2}{2(N+1)}$ & $-\frac{1}{2}$
\end{tabular}

Table 4: Coefficients of the projectors from Eq. (30) for a general $S U(N)$.

The constants $a^{[R]}, b^{[R]}$ and $c^{[R]}$ can be determined using the completeness relation

$$
\sum_{i} \mathbb{P}_{a j, b k}^{\left[R_{i}\right]}=\delta_{a b} \mathbb{1}_{j k}
$$

the projector properties

$$
\mathbb{P}_{a i, b j}^{\left[R_{1}\right]} \mathbb{P}_{b j, c k}^{\left[R_{2}\right]}=\mathbb{P}_{a i, c k}^{\left[R_{1}\right]} \delta_{R_{1} R_{2}}
$$

as well as the proper dimensionality of the representations

$$
\delta_{a b} \delta_{i j} \mathbb{P}_{b j, a i}^{[R]}=d_{R}
$$

The result is given in Tab. 4 and the values for $N=3$ coincide with those of Tab. 2 , The coefficients governing the strength of the potential, $C^{[R]}$, are easily obtained by projecting the amplitude for the one gluon exchange $T_{i j}^{a} F_{b c}^{a}$ onto the three different representations

$$
d_{R} C^{[R]}=\mathbb{P}_{d h, b i}^{[R]} T_{i j}^{a} F_{b c}^{a} \mathbb{P}_{c j, d h}^{[R]},
$$

and the result is listed in Tab. 4.

\section{References}

[1] H. Baer, V. Barger, A. Lessa and X. Tata, JHEP 0909 (2009) 063 arXiv:0907.1922 [hep-ph]].

[2] P. R. Harrison and C. H. Llewellyn Smith, Nucl. Phys. B 213 (1983) 223 [Erratumibid. B 223 (1983) 542].

[3] H. E. Haber and G. L. Kane, Phys. Rept. 117, 75 (1985).

[4] S. Dawson, E. Eichten and C. Quigg, Phys. Rev. D 31 (1985) 1581.

[5] W. Beenakker, R. Höpker, M. Spira and P. M. Zerwas, Nucl. Phys. B 492 (1997) 51 arXiv:hep-ph/9610490. 
[6] W. Beenakker, R. Höpker and M. Spira, arXiv:hep-ph/9611232.

[7] W. Beenakker, M. Krämer, T. Plehn, M. Spira and P. M. Zerwas, Nucl. Phys. B 515 (1998) 3 [arXiv:hep-ph/9710451.

[8] A. Kulesza and L. Motyka, Phys. Rev. Lett. 102 (2009) 111802 arXiv:0807.2405 [hep-ph]].

[9] M. Beneke, P. Falgari, C. Schwinn, Nucl. Phys. B828 (2010) 69. arXiv:0907.1443 [hep-ph]].

[10] U. Langenfeld and S. O. Moch, Phys. Lett. B 675 (2009) 210 arXiv:0901.0802 [hep$\mathrm{ph}]]$.

[11] A. Kulesza and L. Motyka, Phys. Rev. D 80 (2009) 095004 arXiv:0905.4749 [hep$\mathrm{ph}]]$.

[12] W. Beenakker, S. Brensing, M. Krämer, A. Kulesza, E. Laenen and I. Niessen, JHEP 0912, 041 (2009) [arXiv:0909.4418 [hep-ph]].

[13] W. Beenakker, S. Brensing, M. Krämer, A. Kulesza, E. Laenen and I. Niessen, JHEP 1008, 098 (2010) [arXiv:1006.4771 [hep-ph]].

[14] W. Beenakker, S. Brensing, M. Kramer, A. Kulesza, E. Laenen, L. Motyka and I. Niessen, Int. J. Mod. Phys. A 26 (2011) 2637 arXiv:1105.1110 [hep-ph]].

[15] V. S. Fadin, V. A. Khoze and T. Sjöstrand, Z. Phys. C 48, 613 (1990).

[16] K. Hagiwara, Y. Sumino and H. Yokoya, Phys. Lett. B 666, 71 (2008) arXiv:0804.1014 [hep-ph]].

[17] Y. Kiyo, J. H. Kühn, S. Moch, M. Steinhauser and P. Uwer, Eur. Phys. J. C 60 (2009) 375 [arXiv:0812.0919 [hep-ph]].

[18] Y. Sumino and H. Yokoya, JHEP 1009 (2010) 034 [arXiv:1007.0075] [hep-ph]].

[19] K. Hagiwara and H. Yokoya, JHEP 0910 (2009) 049 [arXiv:0909.3204 [hep-ph]].

[20] M. R. Kauth, J. H. Kühn, P. Marquard and M. Steinhauser, Nuclear Physics, Section B 857 (2012), pp. 28 [arXiv:1108.0361 [hep-ph]].

[21] W. Y. Keung and A. Khare, Phys. Rev. D 29, 2657 (1984).

[22] J. H. Kühn and S. Ono, Phys. Lett. B 142, 436 (1984).

[23] J. T. Goldman and H. Haber, Physica 15D, 181 (1985).

[24] V. G. Kartvelishvili, A. V. Tkabladze and E. G. Chikovani, Sov. J. Nucl. Phys. 51, 546 (1990) [Yad. Fiz. 51, 859 (1990)]. 
[25] E. Chikovani, V. Kartvelishvili, R. Shanidze and G. Shaw, Phys. Rev. D 53, 6653 (1996) arXiv:hep-ph/9602249.

[26] M. R. Kauth, J. H. Kühn, P. Marquard and M. Steinhauser, Nucl. Phys. B 831 (2010) 285 [arXiv:0910.2612 [hep-ph]].

[27] Y. Kats and M. D. Schwartz, JHEP 1004 (2010) 016 arXiv:0912.0526 [hep-ph]].

[28] D. Kahawala and Y. Kats, arXiv:1103.3503 [hep-ph].

[29] M. Mühlleitner, A. Djouadi and Y. Mambrini, Comput. Phys. Commun. 168, 46 (2005) arXiv:hep-ph/0311167.

[30] A. Djouadi, J. L. Kneur and G. Moultaka, Comput. Phys. Commun. 176 (2007) 426 arXiv:hep-ph/0211331.

[31] V. S. Fadin and V. A. Khoze, JETP Lett. 46, 525 (1987) [Pisma Zh. Eksp. Teor. Fiz. 46, 417 (1987)].

[32] V. S. Fadin and V. A. Khoze, Sov. J. Nucl. Phys. 48 (1988) 309 [Yad. Fiz. 48 (1988) 487].

[33] T. Collet and M. Steinhauser, arXiv:1107.0530 [hep-ph].

[34] A. D. Martin, W. J. Stirling, R. S. Thorne and G. Watt, Eur. Phys. J. C 63, 189 (2009) arXiv:0901.0002 [hep-ph]].

[35] K. G. Chetyrkin, J. H. Kühn, M. Steinhauser, Comput. Phys. Commun. 133 (2000) 43-65. hep-ph/0004189.

[36] M. Beneke, A. Signer and V. A. Smirnov, Phys. Lett. B 454 (1999) 137 arXiv:hep-ph/9903260.

[37] A. Pineda and A. Signer, Nucl. Phys. B 762 (2007) 67 arXiv:hep-ph/0607239.

[38] A. A. Penin and M. Steinhauser, Phys. Lett. B 538 (2002) 335 arXiv:hep-ph/0204290.

[39] M. Beneke, Y. Kiyo and K. Schuller, Nucl. Phys. B 714, 67 (2005) arXiv:hep-ph/0501289.

[40] A. A. Penin, V. A. Smirnov and M. Steinhauser, Nucl. Phys. B 716 (2005) 303 arXiv:hep-ph/0501042.

[41] A. Bauer, L. Mihaila and J. Salomon, JHEP 0902, 037 (2009) arXiv:0810.5101 [hep-ph]]. 